\title{
A Sharp Partitioning-Inequality for Non-Atomic Probability Measures Based on the Mass of the Infimum of the Measures
}

Theodore P. Hill

School of Mathematics, Georgia Institute of Technology, Atlanta, GA 30332, USA

Summary. If $\mu_{1}, \ldots, \mu_{n}$ are non-atomic probability measures on the same measurable space $(S, \mathscr{F})$, then there is an $\mathscr{F}$-measurable partition $\left\{A_{i}\right\}_{i=1}^{n}$ of $S$ so that $\mu_{i}\left(A_{i}\right) \geqq(n-1+m)^{-1}$ for all $i=1, \ldots, n$, where $m=\left\|\bigwedge_{i=1}^{n} \mu_{i}\right\|$ is the total mass of the largest measure dominated by each of the $\mu_{i}$ 's; moreover, this bound is attained for all $n \geqq 1$ and all $m$ in $[0,1]$. This result is an analog of the bound $(n+1-M)^{-1}$ of Elton et al. [5] based on the mass $M$ of the supremum of the measures; each gives a quantative generalization of a well-known cake-cutting inequality of Urbanik [10] and of Dubins and Spanier [2].

\section{$\S 1$. Introduction}

Suppose $\mu_{1}, \mu_{2}, \ldots, \mu_{n}$ are non-atomic probability measures on the same measurable space $(S, \mathscr{F})$, and let

$$
m=\left\|\bigwedge_{i=1}^{n} \mu_{i}\right\|
$$

denote the total mass of the sub-probability measure $\bigwedge_{i=1}^{n} \mu_{i}$, the largest measure dominated by each of the measures $\mu_{i}$. The main purpose of this note is to prove the following result.

Theorem 1. If $\mu_{1}, \ldots, \mu_{n}$ are non-atomic probability measures on the same measurable space $(S, \mathscr{F})$, then there is an $\mathscr{F}$-measurable partition $\left\{A_{i}\right\}_{i=1}^{n}$ of $S$ satisfying

$$
\mu_{i}\left(A_{i}\right) \geqq(n-1+m)^{-1} \quad \text { for all } i=1, \ldots, n \text {; }
$$

moreover, this bound is attained for all positive integers $n$ and all $m \in[0,1]$. 
Theorem 1 is a direct analog of a sharp partitioning result of Elton et al. [5] based on the total mass $M=\left\|\bigvee_{i=1}^{n} \mu_{i}\right\|$ of the smallest measure $\bigvee_{i=1}^{n} \mu_{i}$ dominating each of the $\mu_{i}$ 's; namely, the existence of a measurable partition $\left\{A_{i}\right\}_{i=1}^{n}$ of $S$ satisfying

$$
\mu_{i}\left(A_{i}\right) \geqq(n+1-M)^{-1} \quad \text { for all } i=1, \ldots, n .
$$

It is easy to see that for $n=2$, both inequalities (1) and (2) are identical (since in that case $m+M=2$ ), but that for $n>2$ neither implies the other. Since

$$
m=1 \Leftrightarrow M=1 \Leftrightarrow \mu_{i}=\mu_{j} \quad \text { for all } i, j=1, \ldots, n
$$

both inequalities (1) and (2) give quantitative generalizations of a well-known "cake-cutting" result of Urbanik [10] and of Dubins and Spanier [2] which state that if $\mu_{i} \neq \mu_{j}$ for some $i \neq j$, then there is a measurable partition $\left\{A_{i}\right\}_{i=1}^{n}$ of $S$ satisfying

$$
\mu_{i}\left(A_{i}\right)>n^{-1} \quad \text { for all } i=1, \ldots, n .
$$

(In the cake-cutting interpretation of these inequalities, $S$ represents a cake which must be divided among $n$ people, and $\mu_{i}(A)$ represents the value of piece $A$ to person $i$; the reader is referred to [2] or [5] for more details.)

\section{§2. Proof of Main Theorem}

Since the conclusion of Theorem 1 is trivial if $n=1$, assume $n>1$. Throughout this section, $\Pi_{k}$ denotes the collection of $\mathscr{F}$-measurable $k$-partitions of $S$, that is

$$
\Pi_{k}=\left\{\left\{A_{i}\right\}_{i=1}^{k}: A_{i} \cap A_{j}=\emptyset \text { if } i \neq j \text {, and } A_{i} \in \mathscr{F} \forall i=1, \ldots, k\right\} \text {, }
$$

and $\vec{\mu}=\left(\mu_{1}, \ldots, \mu_{n}\right)$ is an $n$-dimensional vector-valued measure each of whose coordinates is a non-atomic (non-negative, countably additive) finite measure.

Let $P R(\vec{\mu})$ denote the partition-range of $\ddot{\mu}$, that is,

$$
P R(\vec{\mu})=\left\{\left(a_{1}, \ldots, a_{n}\right) \in \mathbb{R}^{n}: \exists\left\{A_{i}\right\}_{i=1}^{n} \in \Pi_{n} \text { with } \mu_{i}\left(A_{i}\right)=a_{i} \forall i=1, \ldots, n\right\} .
$$

Two of the tools in the proof of Theorem 1 are a generalization of Lyapounov's Convexity Theorem due to Dvoretzky et al. [3] and an application of the convexity theorem by Neyman which solved Fisher's "Problem of the Nile"; both results are recorded here for convenience, and the reader is referred to [2] for more details concerning these and related results.

Lemma $2.1([3]) . P R(\vec{\mu})$ is convex and compact.

Lemma 2.2 ([9]). For each positive integer $k$, there exists a measurable partition $\left\{E_{i}\right\}_{i=1}^{k}$ of $S$ satisfying

$$
\mu_{j}\left(E_{i}\right)=k^{-1} \mu_{j}(S) \quad \text { for all } j=1, \ldots, n \text { and } i=1, \ldots, k .
$$


The other main tool in the proof is an "inversion principle", which allows any small equipartition value $t$ to be transformed into a new large value $t^{\prime}$, and vice versa. For the remainder of this paper $\mu_{1}, \ldots, \mu_{n}$ are probability measures, and $\vec{i}=(1,1, \ldots, 1)$.

Proposition 2.3 (Inversion Principle).

$$
\vec{a} \in P R(\mu) \Rightarrow \vec{a}^{\prime}=(\overrightarrow{1}-\vec{a}) /(n-1) \in P R(\vec{\mu}) .
$$

Proof. Fix $\vec{a}=\left(a_{1}, \ldots, a_{n}\right) \in P R(\vec{\mu})$, and let $\left\{A_{i}\right\}_{i=1}^{n}$ be any element (partition) in $\Pi_{n}$ with $\vec{a}=\left(\mu_{1}\left(A_{1}\right), \ldots, \mu_{n}\left(A_{n}\right)\right)$.

For each $i=1, \ldots, n$, Lemma 2.2 (with $k=n-1$ and $S=A_{i}$ ) implies the existence of an $\mathscr{F}$-measurable $(n-1)$-partition $\left\{A_{i, k}\right\}_{k=1, k \neq i}^{n}$ of $A_{i}$ satisfying

$$
\mu_{j}\left(A_{i, k}\right)=(n-1)^{-1} \mu_{j}\left(A_{i}\right) \quad \forall j=1, \ldots, n, \forall k \neq i, k=1, \ldots, n .
$$

Letting $B_{j}=\bigcup\left\{A_{i, j}: i \neq j, 1 \leqq i \leqq n\right\}$, it follows easily that

$$
\mu_{j}\left(B_{j}\right)=(n-1)^{-1}\left(1-a_{j}\right) \quad \text { for each } j=1, \ldots, n .
$$

Since $\left\{B_{j}\right\}_{j=1}^{n} \in \Pi_{n}$, this implies that $(\overrightarrow{1}-\vec{a}) /(n-1) \in P R(\vec{\mu})$.

Note that $\vec{a}$ small implies $\vec{a}^{\prime}$ is large (and vice versa) and that $\vec{a}^{\prime \prime} \neq \vec{a}$. The useful aspect in this paper is that in general $\vec{a}^{\prime}$ lies outside the convex hull of $\vec{a}$ and the unit coordinate vectors.

Proof of Theorem 1

Letting $\vec{e}_{i}$ denote the $i^{\text {th }}$ unit coordinate vector $(0, \ldots, 0,1,0, \ldots, 0)$ of $\mathbb{R}^{n}$, it is clear (taking $A_{i}=S, A_{j}=\emptyset$ for $j \neq i$ ) that $\vec{e}_{i} \in P R(\vec{\mu})$ for all $i=1, \ldots, n$.

Fix $\vec{a}=\left(a_{1}, \ldots, a_{n}\right) \in P R(\vec{\mu})$, and let

$$
\beta_{i}=a_{i}\left(n-1+\sum_{j=1}^{n} a_{j}\right)^{-1} \quad \text { for } i=1, \ldots, n ;
$$

and

$$
\beta_{n+1}=(n-1)\left(n-1+\sum_{i=1}^{n} a_{i}\right)^{-1} .
$$

Together, Lemma 2.1 (convexity) and Proposition 2.3 imply that

$$
\vec{v}=\sum_{i=1}^{n} \beta_{i} \vec{e}_{i}+\beta_{n+1}(\overrightarrow{1}-\vec{a}) /(n-1) \in P R(\vec{\mu}),
$$

and an easy calculation shows that $\vec{v}=(\alpha, \alpha, \ldots, \alpha) \in \mathbb{R}^{n}$, where $\alpha=(n-1$ $\left.+\sum_{i=1}^{n} a_{i}\right)^{-1}$.

Choosing $\left\{A_{i}\right\}_{i=1}^{n}$ so that $\sum_{i=1}^{n} a_{i}=m+\varepsilon$ (using the compactness conclusion of Lemma 2.1, $\varepsilon$ may even be taken to be zero), establishes the inequality (1). That this bound is attained follows from the next example. 
Example 2.4. For $n>1$ and $m \in[0,1]$, let $(S, \mathscr{F})=([0,1]$, Borels $)$, let $f_{1}:[0,1] \rightarrow \mathbb{R}$ be $2 I_{[0,1 / 2)}$ and $f_{i}=2 m I_{[0,1 / 2)}+2(1-m) I_{[1 / 2,1]}$ for $i=2, \ldots, n$, and define $\mu_{1}, \ldots, \mu_{n}$ on $(S, \mathscr{F})$ by $\mu_{i}(A)=\int_{A} f_{i} d \lambda$. Then $\mu_{1}, \ldots, \mu_{n}$ are non-atomic probability measures on $(S, \mathscr{F})$ with $\left\|\bigwedge_{i=1}^{n} \mu_{i}\right\|=m$, and an easy calculation shows that for every $\mathscr{F}$-partition $\left\{A_{i}\right\}_{i=1}^{n}$ of $[0,1], \min _{i \leqq n} \mu_{i}\left(A_{i}\right) \leqq(n-1+m)^{-1}$, and that
in fact this bound is attained.

Remarks. The idea to use the Dvoretzky-Wald-Wolfowitz result to establish (1) was triggered by a recent proof of (2) by Legut [8] using that same result; Dubins and Spanier [2] used a similar generalization (matrix convexity) to establish (3).

The extremal case $m=0$ in (1) is not completely analogous to the extremal case $M=n$ in (2), since $M=n$ implies the measures have essentially disjoint support and that the optimal-partitioning constant is always 1 in that case, whereas in the $m=0$ case the optimai-partitioning constant may be strictly bigger than $(n-1)^{-1}$ for some $\vec{\mu}$.

The inversion principle and the bound $\left(n-1+\sum a_{i}\right)^{-1}$ in the proof may be of some use when $m$ is not known or easy to calculate, but instead only several partition-vectors $\left(a_{1}, \ldots, a_{n}\right) \in P R(\vec{\mu})$ are known.

If the measures have atoms, convexity and all the inequalities (1)-(3) may fail; analogs of the convexity theorem and (3) based on the maximum atom mass are contained in [4] and [7]. Similarly, if the measures are no longer assumed to be probability measures, again (1)-(3) may fail; [6] contains an analog of (3) based on the total masses of the measures (the constant $n^{-1}$ is replaced by $n^{-1}$ times the harmonic mean of the total masses of the measures).

\section{§3. Applications}

In the classification problem of statistical decision theory, the minimax risk $R\left(\mu_{1}, \ldots, \mu_{n}\right)$ of probability distributions $\mu_{1}, \ldots, \mu_{n}$ can also be expressed (see $[5])$ as

$$
R\left(\mu_{1}, \ldots, \mu_{n}\right)=1-\sup \left\{\min _{i \leqq n} \mu_{i}\left(A_{i}\right):\left\{A_{i}\right\}_{i=1}^{n} \in \Pi_{n}\right\},
$$

so Theorem 1 has the following immediate consequence.

Corollary 1. If $\mu_{1}, \ldots, \mu_{n}$ are non-atomic probability distributions, then the minimax risk in the corresponding classification problem is at most $(n-2+m) /(n-1$ $+m)$, and this bound is best possible.

In [1] and [5], an application of (2) was made to the problem of distributing $k$ indivisible objects to $n$ people via lotteries, and a similar application can be made of (1).

Acknowledgement. The author is grateful to the referee for suggesting emphasis of the inversion principle, and for several other suggestions and comments. 


\section{References}

1. Demko, S., Hill, T.: Equitable distribution of indivisible objects. Preprint (1985)

2. Dubins, L., Spanier, E.: How to cut a cake fairly. Am. Math. Monthly 68, 1-17 (1961)

3. Dvoretzky, A., Wald, A., Wolfowitz, J.: Relations among certain ranges of vector measures. Pacific J. Math. 1, 59-74 (1951)

4. Elton, J., Hill, T.: A generalization of Lyapounov's convexity theorem to measures with atoms. Proc. Am. Math. Soc., 99, 297-304 (1987)

5. Elton, J., Hill, T., Kertz, R.: Optimal-partitioning inequalities for non-atomic probability measures. Trans. Am. Math. Soc. 296, 703-725 (1986)

6. Hill, T.: Equipartitioning the common domain of non-atomic measures. Math. Z. 189, 415-419 (1985)

7. Hill, T.: Partitioning general probability measures. Ann. Prob., to appear (1987)

8. Legut, J.: Inequalities for $\alpha$-optimal partitioning of measurable space. Math. Z., to appear (1987)

9. Neyman, J.: Un théorème d'existence. C.R. Acad. Sci. Paris 222, 843-845 (1946)

10. Urbanik, K.: Quelques théorèmes sur les mesures. Fund. Math. 41, 150-162 (1955) 